\title{
DIAGNÓSTICOS DE ENFERMAGEM PREVALENTES NO PACIENTE INTERNADO COM SEPSE NO CENTRO DE TERAPIA INTENSIVA
}

Cintia Suemy Kagiyama Dutra', Laura Menezes Silveira², Aive Oliva Santos³, Raíssa Pereira', Angelita Maria Stabile ${ }^{4}$

\author{
'Discente de Enfermagem. Universidade de São Paulo. Ribeirão Preto-SP-Brasil. \\ ${ }^{2}$ Enfermeira. EUniversidade de São Paulo. Ribeirão Preto-SP-Brasil. \\ ${ }^{3}$ Enfermeira. Especialista em Terapia Intensiva. Centro Universitário do Triângulo. Ribeirão Preto-SP-Brasil \\ ${ }^{4}$ Enfermeira. Doutora em Ciências. Universidade de São Paulo. Ribeirão Preto-SP-Brasil.
}

RESUMO: O objetivo deste estudo foi identificar os diagnósticos de enfermagem prevalentes nos pacientes internados com sepse, sepse grave ou choque séptico em um Centro de Terapia Intensiva. A população foi constituída pelos pacientes com idade superior a 18 anos, internados no Centro de Terapia Intensiva, de janeiro a dezembro de 2010. As informações foram coletadas do prontuário, de acordo com as características sociodemográficas e clínicas e diagnósticos de enfermagem registrados. No período estudado, foram internados 103 pacientes, sendo que 79,4\% foram a óbito. Os diagnósticos de enfermagem identificados foram: risco de infecção, risco de aspiração, risco para integridade da pele prejudicada, ventilação espontânea prejudicada, troca de gases prejudicada, perfusão tissular ineficaz cardiopulmonar e integridade da pele prejudicada. Em conclusão, espera-se que a identificação dos diagnósticos, presentes nos prontuários de pacientes com sepse, possam contribuir para a assistência de enfermagem a essa clientela.

DESCRITORES: Sepse; Diagnóstico de enfermagem; Unidades de Terapia Intensiva.

\section{PREVALENT NURSING DIAGNOSIS IN PATIENTS HOSPITALIZED WITH SEPSIS AT THE INTENSIVE CARE UNIT}

\begin{abstract}
The objective in this study was to identify the prevalent nursing diagnoses in patients hospitalized with sepsis, severe sepsis or septic shock at an Intensive Care Unit. The population consisted of patients over 18 years of age, hospitalized at the Intensive Care Unit between January and December 2010. The information was collected from the patient histories, according to the sociodemographic and clinical characteristics and nursing diagnoses registered. In the study period, 103 patients were hospitalized, $79.4 \%$ of whom died. The following nursing diagnoses were identified: risk for infection, risk for aspiration, risk for impaired skin integrity, impaired spontaneous ventilation, impaired gas exchange, ineffective cardiopulmonary tissue perfusion and impaired skin integrity. In conclusion, the identification of the diagnoses present in the histories of sepsis patients is expected to contribute to nursing care for these clients. DESCRIPTORS: Sepsis; Nursing diagnosis; Intensive care units.
\end{abstract}

Autor Correspondente:

Angelita Maria Stabile

Universidade de São Paulo

Av. dos Bandeirantes, 3900 - 1404-902 - Ribeirão Preto-SP-Brasil

E-mail: angelita@eerp.usp.br

\section{DIAGNÓSTICOS DE ENFERMERÍA PREVALENTES EN PACIENTE INTERNADO CON SEPSIS EN CENTRO DE TERAPIA INTENSIVA}

RESUMEN: El objetivo de este estudio fue identificar los diagnósticos de enfermería prevalentes en los pacientes internados con sepsis, sepsis grave o choque séptico en un Centro de Terapia Intensiva. La población fue constituida por los pacientes con edad superior a 18 años, internados en el Centro de Terapia Intensiva, de enero a diciembre de 2010. Las informaciones fueron obtenidas del prontuario, de acuerdo con las características sociodemográficas y clínicas, así como diagnósticos de enfermería registrados. En el periodo estudiado, fueron internados 103 pacientes, siendo que $79,4 \%$ fueron a óbito. Los diagnósticos de enfermería identificados fueron: riesgo de infección, riesgo de aspiración, riesgo para integridad de la piel perjudicada, ventilación espontánea perjudicada, cambio de gases perjudicado, perfusión tisular ineficaz cardiopulmonar y integridad de la piel perjudicada. Se concluye que es necesario que la identificación de los diagnósticos, presentes en los prontuarios de pacientes con sepsis, pueda contribuir para la asistencia de enfermería a esa clientela. DESCRIPTORES: Sepsis; Diagnóstico de enfermería; Unidades de Terapia Intensiva. 


\section{INTRODUÇÃO}

A sepse é um grande desafio para os profissionais de saúde que atuam em Centro de Terapia Intensiva (CTI). Esta enfermidade é conhecida por sua agressividade e por ter como ponto de partida uma infecção seguida de perda progressiva da função de diversos órgãos ${ }^{(1)}$.

A resposta inicial do sistema imunológico aos micro-organismos invasores é denominada síndrome da resposta inflamatória sistêmica (SIRS, sigla em inglês). A presença de um agente infeccioso é o principal disparador da SIRS, entretanto pode haver causas não infecciosas incluindo queimaduras, trauma e cirurgias. Quando a SIRS está associada a um quadro infeccioso suspeito ou confirmado, ela é denominada sepse ${ }^{(2)}$. A sepse grave corresponde à sepse associada à disfunção de órgãos, hipotensão e hipoperfusão tecidual. O choque séptico é caracterizado por hipotensão persistente que não melhora após a reposição volêmica, sendo necessária a administração de agentes vasoativos para manutenção da pressão arterial em níveis compatíveis com a vida ${ }^{(2,3)}$.

No Brasil, Estudo Brasileiro de Epidemiologia da Sepse (BASES- sigla em inglês) analisou 1.387 pacientes admitidos em CTI em hospitais de diferentes regiões do país. Do total de 884 pacientes que permaneceram internados, mais de 24 horas, $88,8 \%$ se enquadraram no critério diagnóstico de SIRS, 46,9\% para sepse, 27,3\% para sepse grave e $23 \%$ para choque séptico. A taxa de mortalidade dos pacientes com sepse, sepse grave e choque séptico foi de 16,7\%, 34,4\% e $65,3 \%$, respectivamente ${ }^{(4)}$.

Os profissionais de enfermagem que atuam em CTI convivem diariamente com pacientes com diagnóstico de sepse. Pelo fato de esses profissionais permanecerem à beira do leito, eles devem estar aptos a identificar os sinais e sintomas da sepse e planejar a assistência de enfermagem, de acordo com as necessidades de cuidado ao paciente. Nesse quadro, a atualização e a competência do enfermeiro tornam-se obrigatórias, quando a finalidade é garantir o aprimoramento do cuidado de enfermagem.

A Sistematização da Assistência de Enfermagem (SAE) organiza o trabalho profissional de enfermagem quanto ao método, pessoal e instrumentos, tornando possível a operacionalização do processo de enfermagem, é um instrumento metodológico que orienta o cuidado profissional de enfermagem e a documentação da prática profissional|(5).

O Processo de Enfermagem (PE), base de sustentação da SAE, é constituído por fases ou etapas que envolvem a coleta de dados, a definição dos Diagnósticos de Enfermagem (DE), planejamento, implementação das intervenções de enfermagem e avaliação dos resultados, por meio de uma abordagem voltada à solução de problemas e ao estabelecimento de metas para atingir os melhores resultados ${ }^{(6)}$.

Para o paciente no $\mathrm{CTI}$, dada a sua situação instável e a necessidade de cuidados de enfermagem mais complexos, a assistência de enfermagem sistematizada é necessária, pois facilitará o domínio apurado da técnica, conciliando-o com o cuidado humanizado e holístico. Quanto maior o número de necessidades afetadas do cliente, maior é a necessidade de planejar a assistência, uma vez que a sistematização das ações visa à organização, eficiência e validade da assistência prestada ${ }^{(7-8)}$.

Um dos caminhos para conhecer as necessidades desses pacientes é identificar os DEs prevalentes nessa clientela, dessa forma, poderão ser promovidas as melhores intervenções de enfermagem para os resultados esperados. Portanto, este estudo objetivou identificar os DEs prevalentes, nos pacientes internados no CTI de um hospital de grande porte, com sepse, sepse grave ou choque séptico e verificar a pertinência dos diagnósticos, de acordo com as características clínicas dessas entidades clínicas.

\section{MATERIAIS E MÉTODOS}

A abordagem metodológica quantitativa foi adotada para o desenvolvimento deste estudo cujo delineamento é transversal e de caráter retrospectivo. $\mathrm{O}$ estudo foi desenvolvido em um hospital público de nível terciário do interior do Estado de São Paulo, cujo CTI geral conta com nove leitos. A população foi constituída pelos pacientes com idade superior a 18 anos, internados no $\mathrm{CTI}$ e que desenvolveram sepse, sepse grave ou choque séptico, de janeiro a dezembro de 2010. Foram excluídos os prontuários dos pacientes provenientes da obstetrícia, da emergência e que não tiveram DEs incluídos nos registros. 
$\mathrm{Na}$ instituição onde foi realizado o estudo, o processo de enfermagem é utilizado rotineiramente no cuidado do paciente internado no $\mathrm{CTI}$, sendo que os DEs foram elaborados de acordo com a taxonomia da North American Nursing Diagnosis Association (NANDA) 2007-2008 ${ }^{(9)}$. Apesar de os DEs terem sido elaborados com base na edição da NANDA 2007-2008(9), a discussão do estudo foi realizada utilizando-se como base a NANDA 2009-2011 ${ }^{(10)}$.

As informações coletadas dos prontuários foram agrupadas de acordo com as variáveis sociodemográficas e clínicas (sexo, idade, valor obtido na Escala de Braden, tempo de internação no CTI, tempo de internação hospitalar, provável foco de infecção desencadeador da sepse, presença do diagnóstico de sepse, sepse grave ou choque séptico e DEs registrados pelo enfermeiro responsável no momento da admissão no $\mathrm{CTI}$ ). A análise dos dados foi realizada na forma descritiva, utilizando o programa Microsoft Office Excel 2007 para calcular a frequência, média e mediana das variáveis investigadas no estudo. Os resultados são apresentados em números absolutos e percentuais em tabelas.

Este trabalho foi elaborado de acordo com as diretrizes contidas na Resolução CNS 196/96 para o desenvolvimento de pesquisa com seres humanos e aprovado pelo Comitê de Ética em Pesquisa da Escola de Enfermagem de Ribeirão Preto - USP (Protocolo ${ }^{\circ}$ 1423/2011), sendo dispensado da aplicação do Termo de Consentimento Livre e Esclarecido.

\section{RESULTADOS}

No período de janeiro a dezembro de 2010, foram internados, no CTI do hospital onde o estudo foi realizado, 103 pacientes com diagnóstico de sepse, sepse grave ou choque séptico e que atendiam aos critérios de inclusão. Do total de pacientes, 55 (53,4\%) eram do sexo masculino com faixa etária entre 18 e 86 anos e mediana de 58 anos. Do total de pacientes diagnosticados, 81 (78,6\%) foram a óbito. Dentre os que foram a óbito, 45 eram do sexo masculino (55,6\%), com idade entre 19 e 86 anos e mediana de 58 anos; e 36 eram do sexo feminino $(44,4 \%)$, com idade entre 18 e 85 anos e média de 58 anos.

A distribuição dos diagnósticos de sepse, sepse grave e choque séptico assim como a mortalidade dos pacientes estão descritas na Tabela 1.

Tabela 1 - Distribuição dos diagnósticos de sepse, sepse grave e choque séptico e respectivas mortalidades. Ribeirão Preto-SP-Brasil, 2013

\begin{tabular}{lcc}
\hline Diagnóstico médico & Diagnóstico $-\mathbf{n}^{\mathbf{0}} \mathbf{( \% )}$ & Mortalidade $\mathbf{-} \mathbf{n}^{\mathbf{o}} \mathbf{( \% )}$ \\
\hline Sepse & $23(22,3)$ & $15(18,5)$ \\
\hline Sepse grave & $17(16,5)$ & $11(13,6)$ \\
\hline Choque séptico & $63(61,2)$ & $55(67,9)$ \\
\hline Total & $103(100)$ & $81(100)$ \\
\hline
\end{tabular}

Na Tabela 2, observa-se a distribuição dos focos de infecção que foram os prováveis desencadeadores da sepse com predominância do foco pulmonar. Dos 103 pacientes avaliados, 39 não apresentaram diagnóstico de sepse, sepse grave e choque séptico com definição explícita do foco, tendo de ser esclarecido.

O valor da Escala Braden dos pacientes, ao serem internados no CTI, variou entre 7 e 20, com mediana de 11. Em 11 prontuários, não constava o valor da Escala Braden do paciente na ficha de internação do CTI. O tempo de internação no CTI variou entre 1 e 62 dias, com mediana de 6 dias. O tempo de internação no hospital até o óbito variou de 1 a 184 dias, com mediana de 6 dias,
Tabela 2. Distribuição dos focos de infecção. Ribeirão Preto-SP-Brasil, 2013

\begin{tabular}{lcc}
\hline Foco da infecção & $\mathbf{n}$ & $\mathbf{\%}$ \\
\hline Pulmonar & 37 & 35,9 \\
\hline Abdominal & 08 & 7,8 \\
\hline Urinário & 06 & 5,8 \\
\hline Cateter venoso central & 05 & 4,9 \\
\hline Cardíaco & 02 & 1,9 \\
\hline Cutâneo & 02 & 1,9 \\
\hline Renal & 02 & 1,9 \\
\hline Síndrome de Fournier & 01 & 1,1 \\
\hline A esclarecer & 40 & 38,8 \\
\hline Total & 103 & 100 \\
\hline
\end{tabular}


enquanto o tempo de internação no hospital do estudo até a alta hospitalar variou de 4 a 71 dias, com mediana de 14,5 dias.

A identificação dos DEs pelo enfermeiro foi realizada apenas no primeiro dia de internação no CTI, não havendo reavaliação diária. Para os 103 pacientes, foram documentados 298 DEs em sete categorias diagnósticas diferentes, sendo que alguns diagnósticos se repetiram na avaliação dos pacientes.

De acordo com a prevalência, os seguintes diagnósticos foram identificados: risco de infecção, risco de aspiração, risco para integridade da pele prejudicada, ventilação espontânea prejudicada, troca de gases prejudicada, perfusão tissular ineficaz cardiopulmonar, integridade da pele prejudicada. A prevalência dos diagnósticos, segundo seus domínios, é apresentada na Tabela 3. No Quadro 1, observam-se os DEs e seus fatores relacionados e, na Quadro 2, estão os DEs e suas características definidoras. A porcentagem de DE refere-se à frequência de identificação de cada DE na população do estudo. As porcentagens dos fatores relacionados e características definidoras referem-se à frequência do respectivo DE.

Tabela 3 - Frequência do DE identificado no CTI, descrito de acordo com a taxonomia da NANDA-I. Ribeirão Preto-SP-Brasil, 2013

\begin{tabular}{lc}
\hline Domínio e Diagnóstico de Enfermagem & n (\%) \\
\hline Segurança/proteção & \\
\hline Risco de infecção & $93(31,2)$ \\
\hline Risco de aspiração & $79(26,5)$ \\
\hline Risco para integridade da pele prejudicada & $75(25,2)$ \\
\hline Integridade da pele prejudicada & $06(2)$ \\
\hline Atividade e repouso & \\
\hline Ventilação espontânea prejudicada & $25(8,4)$ \\
\hline Perfusão tissular ineficaz cardiopulmonar & $09(3)$ \\
\hline Eliminação e troca & \\
\hline Troca de gases prejudicada & $11(3,7)$ \\
\hline Total & $298(100)$ \\
\hline
\end{tabular}

Quadro 1 - Frequência dos fatores relacionados dos DEs identificados, descritos de acordo com a NANDA-I. Ribeirão Preto-SP-Brasil, 2013

\begin{tabular}{|l|l|}
\hline Diagnóstico de Enfermagem, $\mathbf{n}(\%)$ & Fatores Relacionados n (\%) \\
\hline Risco de infecção, $93(90,3)$ & $\begin{array}{l}\text { Imunossupressão, 29 (31,1); Procedimentos inva- } \\
\text { sivos, 83 (89,2); Doenças crônicas, 48 (51,6); Defesas } \\
\text { primárias e secundárias inadequadas, 41 (44,1) }\end{array}$ \\
\hline Risco de aspiração, $79(76,7)$ & $\begin{array}{l}\text { Traqueostomia, 64 (81,0); Nível de consciência reduzi- } \\
\text { do, 53 (67); Deglutição prejudicada, 22 (27,8); Alimen- } \\
\text { tação por sondas, 34 (43) }\end{array}$ \\
\hline Risco para integridade da pele prejudicada, 75 (72,8) & $\begin{array}{l}\text { Estado nutricional prejudicado, 31 (41,3); Imobilidade } \\
\text { física, 49 (65,3); Pele úmida, 13 (17,3); Fatores mecâni- } \\
\text { cos, 60 (80) }\end{array}$ \\
\hline Ventilação espontânea prejudicada, 25 (24,3) & $\begin{array}{l}\text { Fadiga da musculatura, 18 (72) } \\
\text { Fatores metabólicos, 09 (36) }\end{array}$ \\
\hline Troca de gases prejudicada, 11 (10,7) & $\begin{array}{l}\text { Desequilíbrio na ventilação-perfusão, 10 (90,9) } \\
\text { Mudança da membrana alveolocapilar, 05 (45,5\%) }\end{array}$ \\
\hline Perfusão tissular ineficaz cardiopulmonar, 09 (8,7) & $\begin{array}{l}\text { Descompensação entre ventilação e fluxo sanguíneo, } \\
\text { 06 (66,7); Transporte prejudicado de O2, 05 (55,6); } \\
\text { Hiper ou hipovolemia, 04 (44,4) }\end{array}$ \\
\hline Integridade da pele prejudicada, 06 (5,8) & $\begin{array}{l}\text { Fatores mecânicos, 06 (100); Pele úmida, 02 (33,3); } \\
\text { Imobilidade física, 04 (66,7); Estado nutricional preju- } \\
\text { dicado, 05 (83,3) }\end{array}$ \\
\hline
\end{tabular}


Quadro 2 - Frequência das características definidoras dos DEs identificados, descritos de acordo com a NANDA-I. Ribeirão Preto-SP-Brasil, 2013

\begin{tabular}{|l|l|}
\hline Diagnóstico de Enfermagem, $\mathbf{n}(\%)$ & Características Definidoras, N (\%) \\
\hline Risco de infecção, $93(90,3)$ & Não se aplica \\
\hline Risco de aspiração, $79(76,7)$ & Não se aplica \\
\hline Risco para integridade da pele prejudicada, $75(72,8)$ & Não se aplica \\
\hline Ventilação espontânea prejudicada, 25 (24,3) & $\begin{array}{l}\text { Agitação, 10 (40); } \\
\text { Dispneia, 14 (56); } \\
\text { Saturação de O2 diminuída, 13 (52); } \\
\text { Uso aumentado da musculatura acessória, 13 (52) }\end{array}$ \\
\hline Troca de gases prejudicada, 11 (10,7) & $\begin{array}{l}\text { Taquipneia/dispneia, 07 (63,6); Gases sanguíneos } \\
\text { arteriais anormais, 06 (54,5); Hipoxemia / hipóxia, 03 } \\
(27,3) ; \text { Alterações respiratórias quanto a ritmo e à pro- } \\
\text { fundidade, 05 (45,5) }\end{array}$ \\
\hline Perfusão tissular ineficaz cardiopulmonar, 09 (8,7) & $\begin{array}{l}\text { Arritmias, 03 (33,3); } \\
\text { Pré-cordialgia, 03 (33,3); } \\
\text { Broncoespasmo, 02 (22,2); } \\
\text { Gases sanguíneos arteriais anormais, 03 (33,3) }\end{array}$ \\
\hline Integridade da pele prejudicada, 06 (5,8) & $\begin{array}{l}\text { Destruição de camadas da pele, 01 (16,7); Rompimen- } \\
\text { to da superfície da pele, 03 (50) }\end{array}$ \\
\hline
\end{tabular}

\section{DISCUSSÃO}

Este estudo identificou os DEs prevalentes em pacientes internados com diagnóstico de sepse, sepse grave e choque séptico, no CTI de um hospital de grande porte, e pode ser uma ferramenta para identificação das necessidades de cuidados dessa clientela.

Apesar de os DEs terem sido elaborados com base na edição da NANDA 2007-2008 ${ }^{(9)}$, a discussão do estudo foi realizada utilizandose como base a NANDA 2009-2011 ${ }^{(10)}$. O DE prevalente foi o de risco de infecção o qual estava relacionado à imunossupressão, a procedimentos invasivos, a doenças crônicas e a defesas primárias e secundárias inadequadas. Este achado indica que pode haver falta de compreensão acerca da definição de sepse e dos DEs, visto que os pacientes com este diagnóstico tinham sido diagnosticados com sepse, sepse grave ou choque séptico, ou seja, já havia infecção instalada e não mais um risco. Por outro lado, alguns diagnósticos, que deveriam estar presentes dadas as características clínicas da sepse, não foram elencados.

A hiperglicemia é um achado comum em pacientes com sepse, considerada um marcador de mau prognóstico para pacientes graves, tanto clínicos quanto cirúrgicos ${ }^{(11-12)}$. Estima-se que de 50 a $85 \%$ dos pacientes internados em CTI apresentem hiperglicemia, entretanto o diagnóstico risco de glicemia instável não foi identificado nos pacientes ${ }^{(13)}$. Outros diagnósticos que certamente estariam presentes nessa clientela, mas que não foram mencionados, são hipertermia e hipotermia ${ }^{(10)}$. Alterações da temperatura corporal como a febre e a hipotermia são achados comuns nos pacientes com sepse $\mathrm{e}^{(14)}$ e estão associadas com aumento dos índices de morbidade e mortalidade, sendo que pacientes com hipotermia têm pior prognóstico do que aqueles com febre ${ }^{(15)}$.

Por outro lado, observa-se que o DE perfusão tissular ineficaz cardiopulmonar estava presente, relacionado à descompensação entre ventilação e fluxo sanguíneo, transporte prejudicado de $\mathrm{O} 2$ e hiper ou hipovolemia e foi manifestado por arritmias, pré-cordialgia, broncoespasmo e gases sanguíneos arteriais anormais. O que caracteriza o quadro de choque séptico é a hipotensão arterial persistente, apesar da ressuscitação volêmica, sendo necessária a administração de agentes vasopressores para a manutenção da pressão arterial em níveis adequados à vida ${ }^{(16)}$. A hipotensão resulta em hipoperfusão tecidual, a qual pode acarretar o desenvolvimento de disfunções de órgãos, principalmente do encéfalo, rins, coração e fígado ${ }^{(17)}$.

Outros DEs elencados e que se mostraram pertinentes foram ventilação espontânea prejudicada e troca de gases prejudicada, Pelo fato desses pacientes estarem gravemente 
doentes, acamados, com rebaixamento do nível de consciência e muitas vezes necessitando de entubação, era de se esperar que eles apresentassem déficit na oxigenação ou eliminação de dióxido de carbono e incapacidade de manter respiração adequada ${ }^{(10)}$. Adicionalmente, o DE risco de aspiração também se mostrou adequado.

Pacientes internados e gravemente enfermos têm maior risco de aspiração de conteúdo gástrico e consequente desenvolvimento de pneumonia aspirativa. Este risco está relacionado a fatores como posição supina, gastroparesia e utilização de narcóticos. Os episódios de aspiração são frequentes em pacientes internados no CTI e cursam com graves consequências, aumentando a sua morbidade. Neste estudo, o DE risco de aspiração estava relacionado à presença de traqueostomia, nível de consciência reduzido, deglutição prejudicada e alimentação por sondas.

Os DEs risco para integridade da pele prejudicada e integridade da pele prejudicada estavam presentes em 81 pacientes e estavam relacionados a fatores mecânicos, pele úmida, imobilidade física e estado nutricional prejudicado. O DE integridade da pele prejudicada tinha como características definidoras a destruição de camadas da pele e o rompimento da superfície da pele. Estes diagnósticos mostram-se extremamente pertinentes frente aos escores de Escala de Braden encontrados.

A Escala de Braden é composta de seis subclasses que refletem o grau de percepção sensorial, umidade, atividade física, nutrição, mobilidade, fricção e cisalhamento ${ }^{(18)}$. Neste estudo, o valor médio da Escala de Braden foi 11 , o que indica que os pacientes possuem risco moderado para o desenvolvimento de úlceras por pressão.

A literatura traz outros estudos em que foram avaliados os DEs em pacientes com sepse, sepse grave e choque séptico ${ }^{(19-20)}$. Apesar de possuírem características metodológicas diferentes e um número reduzido de participantes, observase que os DEs perfusão tissular ineficaz, ventilação espontânea prejudicada e risco para integridade da pele prejudicada também foram elencados. Este fato indica que há similaridade nas características clínicas dos pacientes, independente do local onde o estudo foi realizado.
A mortalidade encontrada neste estudo $(78,64 \%)$ foi maior do que na literatura nacional e internacional. Na Europa, foram encontradas taxas de mortalidade total nos casos de sepse, sepse grave e choque séptico de $27 \%, 32,2 \%$ e $54,1 \%$, respectivamente, e no Brasil 15\%, 34,6\% e $50 \%$, respectivamente ${ }^{(21-22)}$.

A mortalidade elevada encontrada pode ser explicada pelo quadro severo dos pacientes admitidos no CTI. Neste estudo, houve predominância de pacientes com o diagnóstico de choque séptico $(61,2 \%)$, com mortalidade de $67,9 \%$. A literatura aponta para taxas do diagnóstico de choque séptico de 52,1\%, assim como sendo esta a maior causa de mortalidade $(68,96 \%)^{(23)}$. Uma possível explicação para a alta taxa de pacientes com choque séptico pode ser o retardo no diagnóstico da sepse, sendo diagnosticados apenas aqueles pacientes em quadro avançado e que possuíam maior possibilidade de ir a óbito, portanto, a taxa de mortalidade pode ter sido superestimada. Neste estudo, a mediana da idade dos pacientes foi de 58 anos, para ambos os sexos, estando de acordo com estudos prévios que mostram que tanto a incidência quanto a mortalidade por sepse aumentam com a idade ${ }^{(24-25)}$.

O foco de infecção prevalente neste estudo foi o pulmonar, sendo responsável por 35,9\% das infecções. Esta informação está de acordo com a literatura nacional e internacional. Um recente estudo brasileiro mostrou que o foco infeccioso principal de sepse grave e choque séptico foi o trato respiratório, estando presente em 53,8\% dos pacientes ${ }^{(4,26)}$. Os resultados também estão em consonância com estudos internacionais ${ }^{(22)}$.

Algumas das limitações do presente estudo estão relacionadas com seu desenho metodológico. Trata-se de estudo retrospectivo com informações recuperadas de prontuários de pacientes. Assim, é inevitável que algumas condutas tenham sido modificadas, ao longo do período de observação. Adicionalmente, não se tem controle se as informações registradas no prontuário representavam a condição clínica do paciente naquele momento. Acredita-se que um estudo prospectivo, com avaliação diária dos DEs e das intervenções prescritas, poderia fornecer outras informações relevantes sobre o cuidado de enfermagem a essa clientela. 


\section{CONSIDERAÇÕES FINAIS}

A identificação dos DEs presentes nos prontuários de pacientes com sepse pode contribuir para a assistência de enfermagem a essa clientela. Os DEs, se implementados de forma eficaz, fornecem subsídios às ações da equipe de enfermagem direcionadas aos pacientes críticos e, ainda, agem como um elo com a equipe multidisciplinar que auxilia e aprimora os resultados esperados, diminuindo os riscos de complicações e facilitando a implementação das ações. Consequentemente espera-se, com esse cenário, a melhoria da assistência prestada ao paciente.

\section{REFERÊNCIAS}

1. Lagu T, Rothberg MB, Shieh MS, Pekow PS, Steingrub JS, Lindenauer PK. Hospitalizations, costs, and outcomes of severe sepsis in the United States 2003 to 2007. Crit Care Med. 2012;40(3):754-61.

2. Bone RC, Balk RA, Cerra FB, Dellinger RP, Fein AM, Knaus WA, et al. Definitions for sepsis and organ failure and guidelines for the use of innovative therapies in sepsis. The ACCP/SCCM Consensus Conference Committee. American College of Chest Physicians/ Society of Critical Care Medicine. 1992. Chest. 2009;136(5 Suppl):e28.

3. Kumar A, Roberts D, Wood KE, Light B, Parrillo JE, Sharma $S$, et al. Duration of hypotension before initiation of effective antimicrobial therapy is the critical determinant of survival in human septic shock. Crit Care Med. 2006;34(6):1589-96.

4. Sales Júnior JAL, David CM, Hatum R, Souza PCSP, Japiassú A, Pinheiro CTS, et al. Sepse Brasil: estudo epidemiológico da sepse em Unidades de Terapia Intensiva brasileiras. Revista Brasileira de Terapia Intensiva. 2006;18:9-17.

5. Conselho Federal de Enfermagem-COFEN. Resolução COFEN n. 358 de 15 de outubro de 2009. Dispõe sobre a Sistematização da Assistência de Enfermagem - SAE - nas Instituições de Saúde Brasileiras. Rio de Janeiro: Conselho Federal de Enfermagem; 2009.

6. Garcia TR, Nóbrega MML. Processo de enfermagem: da teoria à prática assistencial e de pesquisa. Esc Anna Nery. 2009;13:816-8.

7. Bittar DB, Pereira LV, Lemos RCA. Sistematização da assistência de enfermagem ao paciente crítico: proposta de instrumento de coleta de dados. Texto
Contexto Enferm. 2006;15:617-28.

8. Silva TG, Madureira VSF, Trentini M. Processo de ensino-aprendizagem para implementação do diagnóstico de enfermagem em unidade de terapia intensiva. Cogitare Enferm. 2007;12(3):279-286.

9. North American Nursing Diagnosis-NANDA. Diagnóstico de Enfermagem da NANDA: definições e classificações - 2007/2008. Porto Alegre: Artmed; 2008

10. North American Nursing Diagnosis-NANDA. Diagnósticos de enfermagem da NANDA. Porto Alegre: Artmed; 2010.

11. Leone $M$, Martin C. Vasopressor use in septic shock: an update. Curr Opin Anaesthesiol. 2008;21(2):141-7.

12. Pitrowsky M, Shinotsuka CR, Soares M, Salluh JIF. Controle glicêmico em terapia intensiva 2009: sem sustos e sem surpresas. Rev Bras Ter Intensiva. 2009;21:310-4.

13. Whitcomb BW, Pradhan EK, Pittas AG, Roghmann MC, Perencevich EN. Impact of admission hyperglycemia on hospital mortality in various intensive care unit populations. Crit Care Med. 2005;33(12):2772-7.

14. Blatteis CM. Endotoxic fever: new concepts of its regulation suggest new approaches to its management. Pharmacol Ther. 2006;111(1):194-223.

15. Peres BD, Lopes FF, Melot C, Vincent JL. Body temperature alterations in the critically ill. Intensive Care Med. 2004;30(5):811-6.

16. Jones AE, Puskarich MA. Sepsis-induced tissue hypoperfusion. Crit Care Clin. 2009;25(4):769-79, ix.

17. Abraham E, Singer M. Mechanisms of sepsis-induced organ dysfunction. Crit Care Med. 2007;35(10):240816.

18. Araújo CRD, Lucena STM, Santos IBC, Soares MJGO. A enfermagem e a utilização da escala de Braden em úlcera por pressão. Rev enferm UERJ. 2010;18(3):359364.

19. Neto JMR, Barros MAA, Oliveira MF, Fontes WD, Nóbrega MML. Assistência de enfermagem a pacientes sépticos em uma unidade de terapia intensiva adulta. Rev Ciênc Saúde Nova Esperança. 2011;9(2):17-26. 2011.

20. Neto JMR, Bezerra LM, Barros MAA, Fontes WD. Processo de enfermagem e choque séptico: os cuidados intensivos de enfermagem. Rev enferm UFPE. 2011;5(9):2260-7.

21. Vincent JL, Sakr Y, Sprung CL, Ranieri VM, Reinhart $\mathrm{K}$, Gerlach $\mathrm{H}$, et al. Sepsis in European intensive 
care units: results of the SOAP study. Crit Care Med. 2006;34(2):344-53.

22. Carvalho RHd, Vieira JF, Gontijo Filho PP, Ribas RM. Sepse, sepse grave e choque séptico: aspectos clínicos, epidemiológicos e prognóstico em pacientes de unidade de terapia intensiva de um hospital universitário. Rev Soc Bras Med Trop. 2010;43:591-3.

23. Baracho N, Lopes G, Araujo T, Buissa T, Yano WK. Fatores de risco associados à mortalidade em paciente com sepse grave e choque séptico na unidade de terapia intensiva de um hospital escola do sul de Minas Gerais. Rev Cienc Saude. 2011;1:72-7.

24. Iwashyna TJ, Ely EW, Smith DM, Langa KM. Long-term cognitive impairment and functional disability among survivors of severe sepsis. JAMA. 2010;304(16):178794.

25. Girard TD, Opal SM, Ely EW. Insights into severe sepsis in older patients: from epidemiology to evidencebased management. Clin Infect Dis. 2005;40(5):719-27.

26. Zanon F, Caovilla JJ, Michel RS, Cabeda EV, Ceretta DF, Luckemeyer GD, et al. Sepse na unidade de terapia intensiva: etiologias, fatores prognósticos e mortalidade. Rev Bras Ter Intensiva. 2008;20:128-34. 